Bull. Austral. Math. Soc.

VOL. $72(2005) \quad$ [283-290]

\title{
CHARACTERISATION OF THE ISOMETRIC COMPOSITION OPERATORS ON THE BLOCH SPACE
}

\author{
Flavia Colonna
}

In this paper, we characterise the analytic functions $\varphi$ mapping the open unit disk $\Delta$ into itself whose induced composition operator $C_{\varphi}: f \mapsto f \circ \varphi$ is an isometry on the Bloch space. We show that such functions are either rotations of the identity function or have a factorisation $\varphi=g B$ where $g$ is a non-vanishing analytic function from $\Delta$ into the closure of $\Delta$, and $B$ is an infinite Blaschke product whose zeros form a sequence $\left\{z_{n}\right\}$ containing 0 and a subsequence $\left\{z_{n_{j}}\right\}$ satisfying the conditions $\left|g\left(z_{n_{j}}\right)\right| \rightarrow 1$, and

$$
\lim _{j \rightarrow \infty} \prod_{k \neq n_{j}}\left|\frac{z_{n_{j}}-z_{k}}{1-\overline{z_{n_{j}}} z_{k}}\right|=1 \text {. }
$$

\section{INTRODUCTION}

An analytic function $f$ on $\Delta=\{z \in \mathbb{C}:|z|<1\}$ is said to be Bloch if

$$
\beta_{f}=\sup _{z \in \Delta}\left(1-|z|^{2}\right)\left|f^{\prime}(z)\right|
$$

is finite. Denote by $\mathcal{B}$ the space of all Bloch functions. The map $f \mapsto \beta_{f}$ is a semi-norm on $\mathcal{B}$, and $\mathcal{B}$ is a Banach space, known as the Bloch space, under the norm $\|f\|_{\mathcal{B}}=|f(0)|+\beta_{f}$. We shall refer to these as the Bloch semi-norm and the Bloch norm. For a good reference on Bloch functions, see [1].

By the Schwarz-Pick lemma, if $f$ is an analytic function of $\Delta$ into itself, then for all $z \in \Delta$,

$$
\left(1-|z|^{2}\right)\left|f^{\prime}(z)\right| \leqslant 1-|f(z)|^{2} \leqslant 1,
$$

so $f$ is a Bloch function and $\beta_{f} \leqslant 1$. If, in addition, $f(0)=0$, then $\|f\|_{B} \leqslant 1$. If $S$ is a conformal automorphism of $\Delta$, so that $S=\lambda L_{a}$, where $|\lambda|=1$ and

$$
L_{a}(z)=\frac{a-z}{1-\bar{a} z} .
$$

Received 3rd May, 2005

I wish to dedicate this article to Professor Maurice Heins for his ninetieth birthday. I owe him a debt of gratitude for his great lectures which deeply stimulated my passion for complex analysis. As a thesis advisor, he was always very patient and generous with his time.

Copyright Clearance Centre, Inc. Serial-fee code: 0004-9727/05 \$A2.00+0.00. 
Thus $\left(1-|z|^{2}\right)\left|S^{\prime}(z)\right|=1-|S(z)|^{2}$, so that $\left(1-|a|^{2}\right)\left|S^{\prime}(a)\right|=1$, hence $\beta_{S}=1$. Furthermore, for all $f \in \mathcal{B}$, we have

$$
\left(1-|z|^{2}\right)\left|f^{\prime}(S(z))\right|\left|S^{\prime}(z)\right|=\left(1-|S(z)|^{2}\right)\left|f^{\prime}(S(z))\right|,
$$

then

$$
\sup _{z \in \Delta}\left(1-|z|^{2}\right)\left|f^{\prime}(S(z))\right|\left|S^{\prime}(z)\right|=\sup _{w \in \Delta}\left(1-|w|^{2}\right)\left|f^{\prime}(w)\right|,
$$

that is, $\beta_{f \circ S}=\beta_{f}$. If, in addition, $S(0)=0$ then $S$ is a rotation of the identity: $S=\lambda$ id, for some constant $\lambda$ of modulus 1 .

RemarK 1. By the Schwarz-Pick lemma, for any Bloch function $f$ and any analytic function $\varphi$ mapping $\Delta$ into itself $\beta_{f \circ \varphi} \leqslant \beta_{f}$, and the inequality is actually an equality if $\varphi$ is a conformal automorphism of $\Delta$.

An analytic function $\varphi$ from $\Delta$ into itself induces on $\mathcal{B}$ the bounded composition operator $C_{\varphi}(f)=f \circ \varphi$. The norm of $C_{\varphi}$ is defined by

$$
\left\|C_{\varphi}\right\|_{\mathcal{B}}=\sup _{f \in \mathcal{B},\|f\|_{\mathcal{B}}=1}\|f \circ \varphi\|_{\mathcal{B}}
$$

In [7], Xiong gave estimates for the norm of the composition operator on the Bloch space and obtained several necessary conditions for this operator to be isometric (that is, preserving the Bloch norm). He proved the following result (see [7, Lemma 3 and Theorem 3]):

THEOREM 1. ([7]) Let $\varphi$ be an analytic function from $\Delta$ into itself such that $C_{\varphi}$ is isometric on the Bloch space. Then $\varphi(0)=0$ and $\|\varphi\|_{\mathcal{B}}=1$. In addition, if $\varphi^{\prime}$ is bounded or $\varphi$ is univalent, then $\varphi$ must be a rotation of the identity. If $\varphi$ is not a rotation of the identity, then for each $a \in \Delta$ there exists a sequence $\left\{z_{n}\right\}$ such that $\left|z_{n}\right| \rightarrow 1$ and $\varphi\left(z_{n}\right) \rightarrow a$.

Xiong then raised the following question: Does there exist a function $\varphi$ other than a rotation of the identity function whose corresponding composition operator is isometric on the Bloch space?

In this article, we prove that the answer is affirmative. In section 2 , we characterise the class of all functions $\varphi$ mapping $\Delta$ into itself satisfying the conditions $\varphi(0)=0$ and $\|\varphi\|_{\mathcal{B}}=1$, thus providing a large class of functions other than the rotations of the identity that are candidates for inducing an isometric composition operator on $\mathcal{B}$. We then give a convergence theorem for Bloch functions that will allow us to prove in section 3 (Theorem 5) that, in fact, every analytic function $\varphi$ of $\Delta$ into itself mapping 0 to 0 and having unitary Bloch norm induces an isometric composition operator on the Bloch space. We conclude the paper with an example. 


\section{The analytic Self maps of unitary Bloch Semi-norm}

In [3, Theorem 2], we proved the following result.

THEOREM 2. Let $\varphi$ be an analytic function from $\Delta$ into itself such that $\beta_{\varphi}=1$. Then either $\varphi$ is a conformal automorphism of $\Delta$ or else there exists a sequence $\left\{S_{n}\right\}$ of conformal automorphisms of $\Delta$ such that $\left|S_{n}(0)\right|$ approaches 1 and $\left\{\varphi \circ S_{n}\right\}$ converges to the identity function uniformly on compact subsets of $\Delta$.

To better understand the nature of the functions $\varphi$ described in Theorem 2 which are not conformal automorphisms of $\Delta$, we recall the definition of Blaschke product (see for example, [5, pp. 54-55]).

Let $\left\{z_{n}\right\}$ be a sequence in $\Delta$ such that $\sum\left(1-\left|z_{n}\right|\right)<\infty$. A Blaschke product is an analytic function mapping $\Delta$ into itself of the form

$$
B(z)=z^{m} \prod \frac{\overline{z_{n}}}{\left|z_{n}\right|}\left(\frac{z_{n}-z}{1-\overline{z_{n}} z}\right), z \in \Delta,
$$

where the product is taken over the $z_{n} \neq 0$ and $m$ is the number of terms in the sequence that are equal to 0 . If the zero set is finite (respectively, infinite), $B$ is said to be a finite (respectively, infinite) Blaschke product. In the finite case, the number of zeros (counted according to multiplicity) is called the degree of $B$.

In [3], we showed that the Bloch semi-norm of every finite Blaschke product of degree greater than 1 is strictly less than 1 and in Theorem 4 we characterised the infinite Blaschke products whose Bloch semi-norm is unitary. More generally, we have

THEOREM 3. Let $\varphi$ be an analytic function from $\Delta$ into itself such that $\beta_{\varphi}=1$. Then either $\varphi$ is a conformal automorphism of $\Delta$, or the zeros of $\varphi$ form an infinite sequence $\left\{z_{n}\right\}$ such that

$$
\limsup _{n \rightarrow \infty}\left(1-\left|z_{n}\right|^{2}\right)\left|\varphi^{\prime}\left(z_{n}\right)\right|=1 .
$$

The proof uses the same methods used in the proof of Theorem 4 in [3] for the special case of Blaschke products, but we present it here for completeness.

Proof: Assume $\varphi$ is not a conformal automorphism of $\Delta$. By Theorem 2, there exists a sequence of conformal automorphisms $\left\{S_{n}\right\}$ of $\Delta$ such that $\varphi \circ S_{n}$ converges uniformly to the identity on compact subsets of $\Delta$. If there were some positive number $r$ such that for all $n$ sufficiently large, $\varphi \circ S_{n}$ had no zero of modulus less than $r$, then $1 /\left(\varphi \circ S_{n}\right)$ would converge uniformly to the reciprocal of the identity on a compact neighbourhood of zero, which is obviously impossible. Thus, passing to a subsequence, if necessary, we may assume that $\varphi \circ S_{n}$ has a zero $\zeta_{n}$ such that the sequence $\left\{\zeta_{n}\right\}$ tends to 0 . Let $z_{n}=S_{n}\left(S_{n}\right)$, which is a zero of $\varphi$, and define the sequence of automorphisms $T_{n}=S_{n}^{-1} \circ L_{z_{n}}$. Since $T_{n}(0)=S_{n}^{-1}\left(z_{n}\right)=\zeta_{n} \rightarrow 0$, passing again to a subsequence if necessary, we may assume that $T_{n}(z) \rightarrow \lambda z$ uniformly on compact subsets of $\Delta$, where $\lambda$ 
is some unimodular constant. Thus $\varphi \circ L_{z_{n}}(z)=\varphi \circ S_{n} \circ T_{n}(z) \rightarrow \lambda z$ locally uniformly in $\Delta$. Taking the derivative of $\varphi \circ L_{z_{n}}$ at zero, we obtain $\varphi^{\prime}\left(z_{n}\right)\left(\left|z_{n}\right|^{2}-1\right) \rightarrow \lambda$ as $n \rightarrow \infty$. Hence $\left(1-\left|z_{n}\right|^{2}\right)\left|\varphi^{\prime}\left(z_{n}\right)\right| \rightarrow 1$ as $n \rightarrow \infty$.

OBSERVATION 1. Let $B$ be a Blaschke product with zeros $\left\{z_{n}\right\}_{n \in N}$ and fix $k \in \mathbb{N}$. Then $B$ can be factored as $L_{z_{k}} B_{1}$, where $B_{1}$ is a rotation of the Blaschke product whose zeros are the same as those of $B$, except, possibly, for $z_{k}$. Then

$$
B_{1}\left(z_{k}\right)=\frac{B^{\prime}\left(z_{k}\right)}{L_{z_{k}}^{\prime}\left(z_{k}\right)}=\left(\left|z_{k}\right|^{2}-1\right) B^{\prime}\left(z_{k}\right)
$$

Taking the modulus, we obtain

$$
\left(1-\left|z_{k}\right|^{2}\right)\left|B^{\prime}\left(z_{k}\right)\right|=\left|B_{1}\left(z_{k}\right)\right|=\prod_{n \neq k}\left|\frac{z_{n}-z_{k}}{1-\overline{z_{n}} z_{k}}\right| \text {. }
$$

Since $\left(z_{n}-z_{k}\right) /\left(1-\overline{z_{n}} z_{k}\right)$ and $\left(z_{k}-z_{n}\right) /\left(1-\overline{z_{k}} z_{n}\right)$ have the same modulus, we deduce that

$$
\left(1-\left|z_{k}\right|^{2}\right)\left|B^{\prime}\left(z_{k}\right)\right|=\prod_{n \neq k}\left|\frac{z_{k}-z_{n}}{1-\overline{z_{k}} z_{n}}\right|
$$

Recall that, by the Canonical Factorisation Theorem in $H^{\infty}(\Delta)$, any bounded analytic function $\varphi$ mapping $\Delta$ into itself has the form $g B$, where $g$ is a non-vanishing analytic function of $\Delta$ into the closure of $\Delta$, and $B$ is a Blaschke product.

COROLlary 1. Let $\varphi$ be an analytic function from $\Delta$ into itself. Then $\beta_{\varphi}=1$ if and only if $\varphi$ is a conformal automorphism of $\Delta$, or the zeros of $\varphi$ form an infinite sequence $\left\{z_{n}\right\}$ containing a subsequence $\left\{z_{n_{j}}\right\}$ such that $\left|g\left(z_{n_{j}}\right)\right| \rightarrow 1$ and

$$
\lim _{j \rightarrow \infty} \prod_{k \neq n_{j}}\left|\frac{z_{n_{j}}-z_{k}}{1-\overline{z_{n_{j}}} z_{k}}\right|=1
$$

where $g$ is the non-vanishing analytic function such that $\varphi / g$ is a Blaschke product.

PROOF: Assume that $\beta_{\varphi}=1$ and $\varphi$ is not a conformal automorphism of $\Delta$. We now show that (2) holds for some (necessarily infinite) sequence of zeros of $\varphi$ and the modulus of the nonvanishing factor of the canonical factorisation of $\varphi$ approaches 1 along this sequence. By Theorem 3 , the zeros of $\varphi$ form an infinite sequence $\left\{z_{n}\right\}$ such that $\limsup \left(1-\left|z_{n}\right|^{2}\right)\left|\varphi^{\prime}\left(z_{n}\right)\right|=1$. Thus, there exists a subsequence $\left\{z_{n_{k}}\right\}$ such that $\left(1-\left|z_{n_{k}}^{n \rightarrow \infty}\right|^{2}\right)\left|\varphi^{\prime}\left(z_{n_{k}}\right)\right| \rightarrow 1$ as $k \rightarrow \infty$. By the Canonical Factorisation Theorem, we may write $\varphi=g B$, where $g$ is a nonvanishing analytic function of $\Delta$ into itself, and $B$ is an infinite Blaschke product sharing the zeros of $\varphi$. Using the fact that $B\left(z_{n_{k}}\right)=0$ and (1), we obtain

$$
\left(1-\left|z_{n_{k}}\right|^{2}\right)\left|\varphi^{\prime}\left(z_{n_{k}}\right)\right|=\left(1-\left|z_{n_{k}}\right|^{2}\right)\left|g\left(z_{n_{k}}\right)\right|\left|B^{\prime}\left(z_{n_{k}}\right)\right|=\left|g\left(z_{n_{k}}\right)\right| \prod_{n \neq n_{k}}\left|\frac{z_{n_{k}}-z_{n}}{1-\overline{z_{n_{k}}} z_{n}}\right| .
$$


This implies that

$$
\lim _{k \rightarrow \infty}\left|g\left(z_{n_{k}}\right)\right| \prod_{n \neq n_{k}}\left|\frac{z_{n_{k}}-z_{n}}{1-\overline{z_{n_{k}}} z_{n}}\right|=1 .
$$

Since each factor in the above product is bounded and no greater than 1 , there must be a subsequence $\left\{z_{n_{k_{j}}}\right\}$ of $\left\{z_{n_{k}}\right\}$ such that $\left|g\left(z_{n_{k_{j}}}\right)\right| \rightarrow 1$ as $j \rightarrow \infty$. By (3), we obtain

$$
\lim _{j \rightarrow \infty}\left|g\left(z_{n_{k_{j}}}\right)\right| \prod_{n \neq n_{k_{j}}}\left|\frac{z_{n_{k_{j}}}-z_{n}}{1-\overline{z_{n_{k_{j}}}} z_{n}}\right|=1 .
$$

Thus $\prod_{n \neq n_{k_{j}}}\left|\left(z_{n_{k_{j}}}-z_{n}\right) /\left(1-\overline{z_{n_{k_{j}}}} z_{n}\right)\right|$ must also converge to 1 as $j \rightarrow \infty$, proving (2).

Let us now prove the converse. If $\varphi$ is a conformal automorphism of $\Delta$, as observed in the introduction, its semi-norm is 1 and we are done. So assume $\varphi=g B$ with $g$ nonvanishing analytic function and $B$ Blaschke product with an infinite number of zeros $\left\{z_{n}\right\}$ such that (2) holds for some subsequence $\left\{z_{n_{k}}\right\}$ such that $\left|g\left(z_{n_{k}}\right)\right| \rightarrow 1$. Then

$$
\lim _{k \rightarrow \infty}\left(1-\left|z_{n_{k}}\right|^{2}\right)\left|\varphi^{\prime}\left(z_{n_{k}}\right)\right|=\lim _{k \rightarrow \infty}\left|g\left(z_{n_{k}}\right)\right| \prod_{n \neq n_{k}}\left|\frac{z_{n_{k}}-z_{n}}{1-\overline{z_{n_{k}}} z_{n}}\right|=1
$$

Hence

$$
1=\lim _{k \rightarrow \infty}\left(1-\left|z_{n_{k}}\right|^{2}\right)\left|\varphi^{\prime}\left(z_{n_{k}}\right)\right| \leqslant b_{\varphi} \leqslant 1
$$

proving that $\varphi$ has semi-norm 1 .

Recall that an infinite sequence $\left\{z_{n}\right\}$ in $\Delta$ is said to be interpolating if for each bounded sequence $\left\{w_{n}\right\}$ in $\mathbb{C}$ there exists a bounded analytic function mapping each $z_{n}$ to $w_{n}$. Carleson [2] proved that an infinite sequence $\left\{z_{n}\right\}$ in $\Delta$ is interpolating if and only if

$$
\inf _{n} \prod_{k \neq n}\left|\frac{z_{n}-z_{k}}{1-\overline{z_{n}} z_{k}}\right|>0
$$

An interpolating sequence $\left\{z_{n}\right\}$ is said to be thin if

$$
\prod_{k \neq n}\left|\frac{z_{n}-z_{k}}{1-\overline{z_{n}} z_{k}}\right| \rightarrow 1 \text { as } n \rightarrow \infty
$$

As a consequence of Corollary 1 we deduce that the zero set of an analytic function $\varphi$ of $\Delta$ into itself, not a rotation of the identity, with Bloch semi-norm one must contain a thin sequence, but the full sequence of the zeros of $\varphi$ need not be interpolating.

We now recall a convergence theorem for Bloch functions (see [4, Theorem 11] for details). This result was based on the fact that the Bloch functions are precisely the Lipschitz maps between the Poincaré disk and the complex plane under the Euclidean 
distance. Furthermore, the Lipschitz number of a Bloch function $f$ is precisely its Bloch semi-norm $\beta_{f}$, that is,

$$
\beta_{f}=\sup _{z \neq w} \frac{|f(z)-f(w)|}{\rho(z, w)},
$$

where $\rho$ is the hyperbolic distance in $\Delta$ :

$$
\rho(z, w)=\frac{1}{2} \log \left(\frac{1+\left|L_{z}(w)\right|}{1-\left|L_{z}(w)\right|}\right), \text { for } z, w \in \Delta .
$$

THEOREM 4. ([4]) Let $\left\{f_{n}\right\}$ be a sequence of Bloch functions converging locally uniformly to some analytic function $f$. If the sequence $\left\{\beta_{f_{n}}\right\}$ is bounded, then $f$ is Bloch and

$$
\beta_{f} \leqslant \liminf _{n \rightarrow \infty} \beta_{f_{n}} .
$$

That is, the function $f \mapsto \beta_{f}$ is lower semi-continuous on $\mathcal{B}$.

OBSERVATION 2. An example of an infinite Blaschke product with unitary Bloch seminorm was constructed by Heins in [6] and referred to as a universal Blaschke product. He proved the existence of an infinite Blaschke product $b$ satisfying the following properties:

(a) 1 is the only cluster point of the zeros of $b$, and

(b) there exists an increasing sequence $\left\{x_{n}\right\}$ converging to 1 , with $x_{0}=0$, such that every analytic function $g$ mapping $\Delta$ into itself can be expressed as the uniform limit in $\Delta$ of $g_{k}=b \circ L_{x_{n(k)}} \circ(-\mathrm{id})$ for some subsequence $\left\{x_{n(k)}\right\}$.

To verify that $\beta_{b}=1$ observe that by Theorem 4 , we have

$$
1=\beta_{\text {id }} \leqslant \liminf _{k \rightarrow \infty} \beta_{g_{k}}=\beta_{b} \leqslant 1 .
$$

We shall use a universal Blaschke product to obtain an example of a function $\varphi$, not a rotation of the identity, such that $C_{\varphi}$ is an isometry of the Bloch space.

\section{ANALYTIC SELF MAPS OF $\triangle$ INDUCING AN ISOMETRY ON $\mathcal{B}$}

In this section, we characterise the analytic self maps of $\Delta$ inducing an isometry on $\mathcal{B}$. In the following lemma, we show that instead of having to test a composition operator on all Bloch functions, it suffices to restrict our attention to the Bloch functions that have 0 as a fixed point.

LEMMA 1. Let $\varphi$ be an analytic function of $\Delta$ into itself such that $\varphi(0)=0$ and $\|\varphi\|_{\mathcal{B}}=1$. Suppose that for all $g \in \mathcal{B}$ such that $g(0)=0$ and $\|g\|_{\mathcal{B}}=1$, we have $\|g \circ \varphi\|_{\mathcal{B}}=1$. Then $C_{\varphi}$ is isometric on the Bloch space.

Proof: Assume $f \in \mathcal{B}$ and $\|f\|_{\mathcal{B}}=1$. Let $f(0)=a$ and observe that $|a| \leqslant 1$ since $|f(0)| \leqslant\|f\|_{B}$. 
If $|a|=1, \beta_{f}=\|f\|_{B}-|a|=0$, so $f^{\prime}$ must be identically zero, hence $f$ is a constant. Then $f \circ \varphi$ is a constant as well and so $\|f \circ \varphi\|_{B}=|f(\varphi(0))|=|f(0)|=1$.

So assume $|a|<1$. Define the analytic function $g=(f-a) /(1-|a|)$. Then $g(0)=0$ and

$$
\sup _{z \in \Delta}\left(1-|z|^{2}\right)\left|g^{\prime}(z)\right|=\sup _{z \in \Delta} \frac{\left(1-|z|^{2}\right)\left|f^{\prime}(z)\right|}{1-|a|}=\frac{\beta_{f}}{1-|a|}=\frac{\|f\|_{B}-|a|}{1-|a|}=1 .
$$

Thus $g \in \mathcal{B}$ and $\|g\|_{\mathcal{B}}=\beta_{g}=1$. By our hypothesis, $\|g \circ \varphi\|_{\mathcal{B}}=1$, so

$$
1=\beta_{g \circ \varphi}=\frac{1}{1-|a|} \beta_{f \circ \varphi}=\frac{\|f \circ \varphi\|_{B}-|a|}{1-|a|} .
$$

Hence $\|f \circ \varphi\|_{\mathcal{B}}=1$, completing the proof.

We are now ready to prove our main result.

THEOREM 5. The analytic functions $\varphi$ from $\Delta$ into itself which induce an isometric composition operator on the Bloch space are precisely the functions mapping 0 to 0 and having Bloch semi-norm equal to one.

Proof: The necessity follows from Theorem 1. To prove the sufficiency, assume $\varphi$ satisfies the conditions $\varphi(0)=0$ and $\beta_{\varphi}=1$. By Theorem 2, either $\varphi$ is a rotation of the identity, in which case the corresponding composition operator is clearly isometric on $\mathcal{B}$, or there exists a sequence $\left\{S_{n}\right\}$ of conformal automorphisms of $\Delta$ such that $\left|S_{n}(0)\right|$ approaches 1 and $\left\{\varphi \circ S_{n}\right\}$ converges to the identity function uniformly on compact subsets of $\Delta$. We now show that $\varphi$ induces an isometric composition operator on $\mathcal{B}$. By Lemma 1, it suffices to show that if a Bloch function $f$ satisfies $f(0)=0$ and $\beta_{f}=1$, then $\beta_{f \circ \varphi}=1$. Since $\lim _{n \rightarrow \infty} \varphi \circ S_{n}(z)=z$ locally uniformly in $\Delta$, the sequence $\left\{f \circ \varphi \circ S_{n}\right\}$ converges to $f$ uniformly on compact subsets of $\Delta$, and $\beta_{f \circ \varphi \circ S_{n}}$ is bounded above by 1 , so by Theorem 4 ,

$$
\beta_{f} \leqslant \liminf _{n \rightarrow \infty} \beta_{f \circ \varphi \circ S_{n}} .
$$

On the other hand, by Remark 1 , for each $n, \beta_{f \circ \varphi \circ S_{n}} \leqslant \beta_{f}$, so the latter inequality is actually an equality. Since $\beta_{f \circ \varphi \circ S_{n}}=\beta_{f \circ \varphi}$, for each $n \in \mathbb{N}$, it follows that $\beta_{f \circ \varphi}=\beta_{f}=1$, proving the result.

As a direct application of Theorem 5 and Corollary 1, we deduce the following result.

COROLlaRY 2. The analytic functions $\varphi$ from $\Delta$ into itself which induce an isometric composition operator on the Bloch space are precisely the rotations of the identity and the functions of the form $\varphi=g B$ where $g$ is a nonvanishing analytic function of $\Delta$ into the closure of $\Delta$, and $B$ is a Blaschke product whose zeroes form an infinite sequence $\left\{z_{n}\right\}$ containing 0 and an infinite subsequence $\left\{z_{n_{j}}\right\}$ such that $\left|g\left(z_{n_{j}}\right)\right| \rightarrow 1$ and

$$
\lim _{j \rightarrow \infty} \prod_{k \neq n_{j}}\left|\frac{z_{n_{j}}-z_{k}}{1-\overline{z_{n_{j}}} z_{k}}\right|=1
$$


EXAMPLE 3.1. Let $b$ be a Blaschke product satisfying conditions (a) and (b) of Observation 2 . In particular, $b$ has infinitely many zeros, and $\beta_{b}=1$. Let $a$ be a zero of $b$. Then the function $\varphi=b \circ L_{a}$ is a unimodular constant multiple of an infinite Blaschke product mapping 0 to 0 such that $\beta_{\varphi}=1$. By Theorem $5, C_{\varphi}$ is isometric on the Bloch space.

\section{REFERENCES}

[1] J.M. Anderson, J. Clunie and Ch. Pommerenke, 'On Bloch functions and normal functions', J. Reine Angew. Math. 279 (1974), 12-37.

[2] L. Carleson, 'An interpolation problem for bounded analytic functions', Amer. J. Math. 80 (1958), 921-930.

[3] F. Colonna, 'The Bloch constant of bounded analytic functions', J. London Math. Soc. (2) 36 (1987), 95-101.

[4] F. Colonna, 'Bloch and normal functions and their relation', Rend. Circ. Mat. Palermo (2) 38 (1989), 161-180.

[5] J.B. Garnett, Bounded analytic functions (Acadademic Press, New York, 1981).

[6] M.H. Heins, 'A universal Blaschke product', Arch. Math. 6 (1955), 41-44.

[7] C. Xiong, 'Norm of composition operators on the Bloch space', Bull. Austral. Math. Soc. 70 (2004), 293-299.

Department of Mathematics

George Mason University

Fairfax, VA 22030-4444

United States of America

e-mail: fcolonna@gmu.edu 